\title{
Barrier Function-Based Adaptive Integral Sliding Mode Finite-time Attitude Control for Rigid Spacecraft
}

Jie Wang ( $\nabla$ wangjie@hebut.edu.cn )

University of Science and Technology Beijing https://orcid.org/0000-0002-8613-3976

YuShang Hu

Hebei University of Technology

Wenqiang Ji

Hebei University of Technology

\section{Research Article}

Keywords: Barrier functions, Integral sliding mode control, Rigid spacecraft, Attitude control, Finitetime control

Posted Date: June 16th, 2021

DOI: https://doi.org/10.21203/rs.3.rs-596811/v1

License: (c) (i) This work is licensed under a Creative Commons Attribution 4.0 International License. Read Full License 


\section{Abstract}

This paper investigates the problem of the finite-time attitude tracking control for rigid spacecrafts with external disturbances and inertia uncertainties. Firstly, a finite-time approach is designed to achieve attitude tracking control of the rigid spacecraft in absence of disturbances and inertia uncertainties and the time of convergence can be chosen in advance. Then, the integral sliding mode combined with barrier function-based adaptive laws is proposed to reject the disturbances and inertia uncertainties, and at the same time, a barrier function-based adaptive method can also ensure the solutions of the rigid spacecraft system belonging to a stipulated vicinity of the intended variables starting from the initial moment and the uncertainties' upper bound is not overestimated. Finally, numerical simulation is provided to illustrate the efficiency of the proposed control protocol.

\section{Full Text}

This preprint is available for download as a PDF.

\section{Figures}

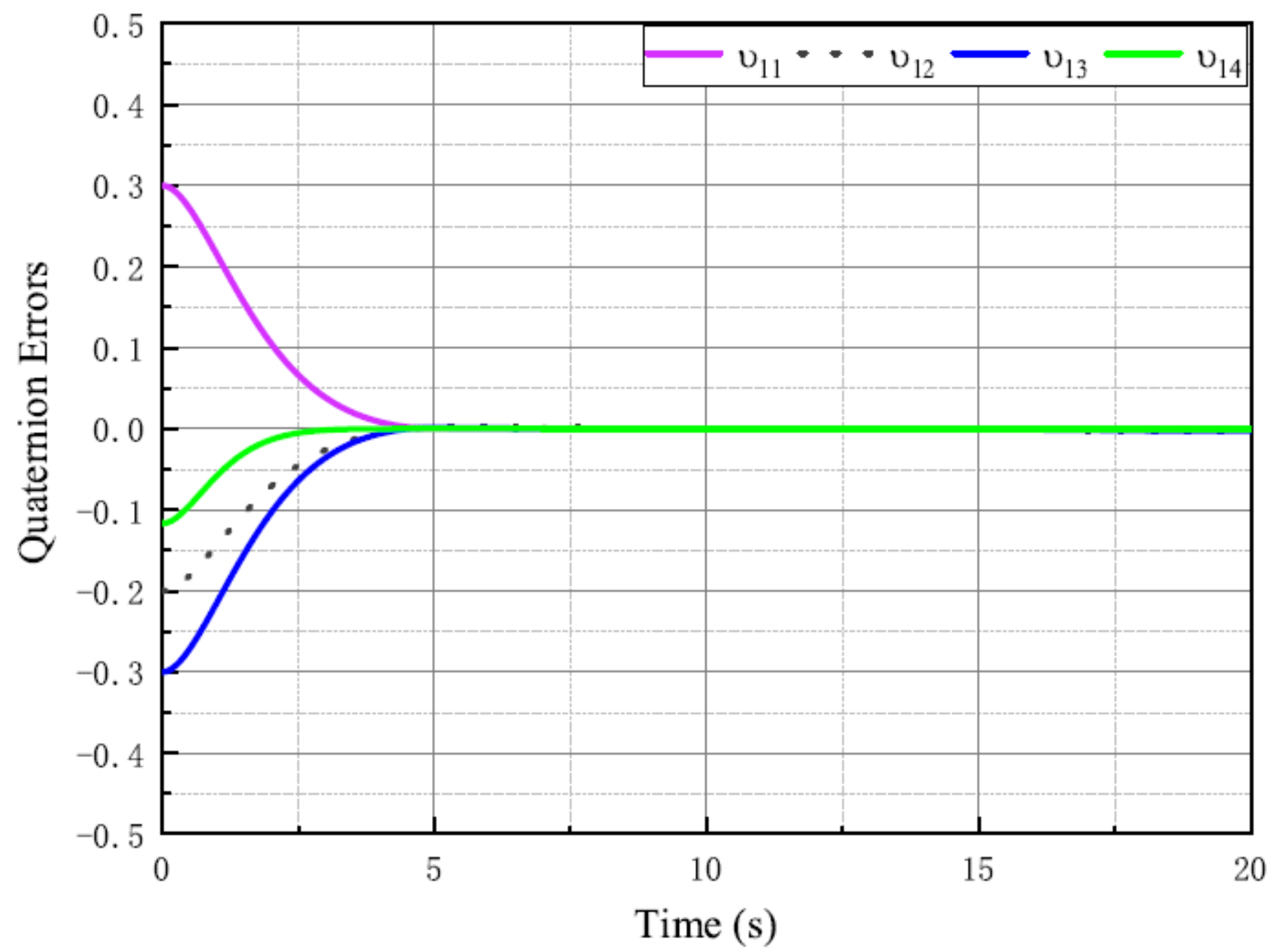


Figure 1

Attitude quaternion tracking errors

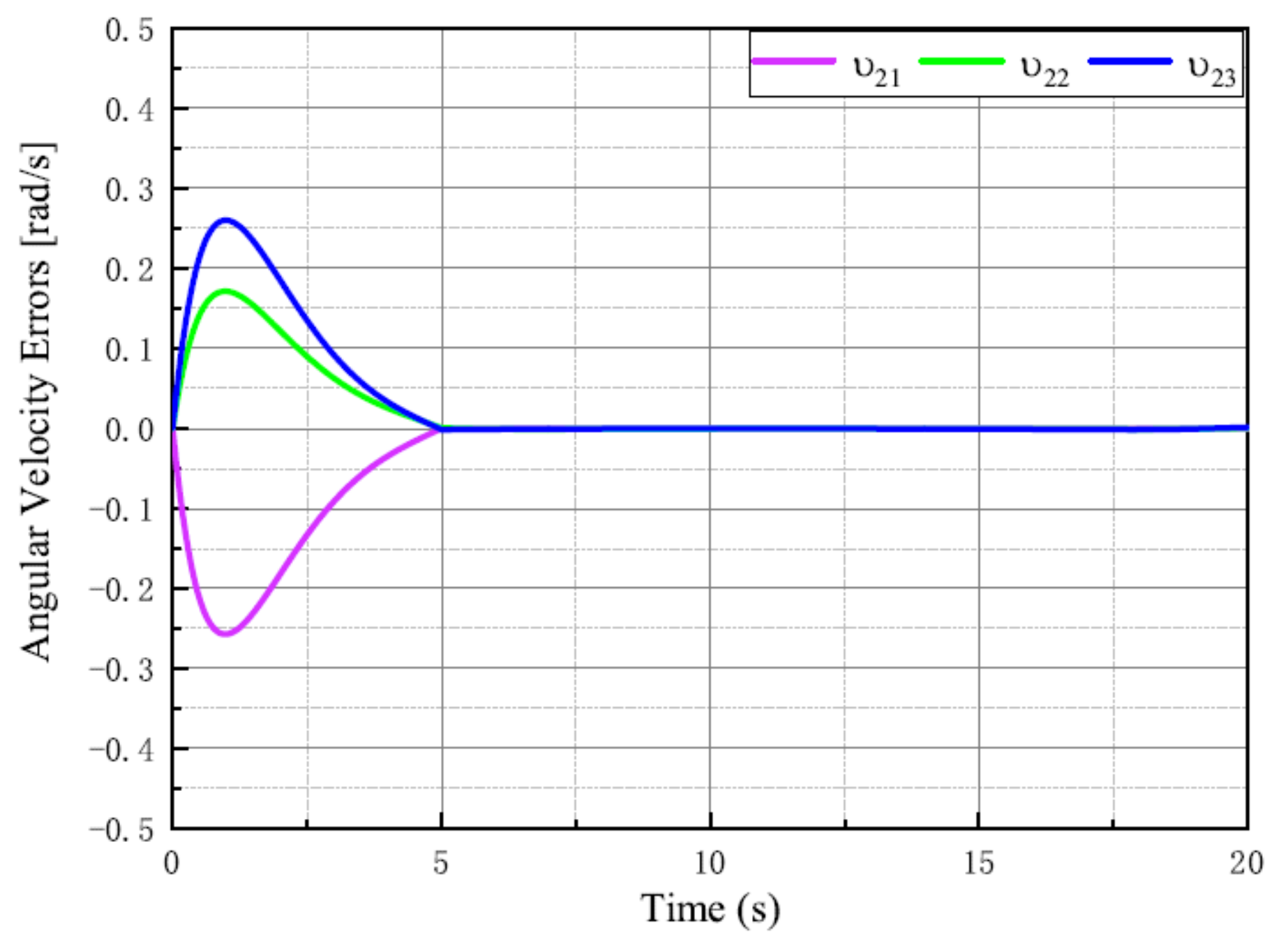

Figure 2

Angular velocity tracking errors 


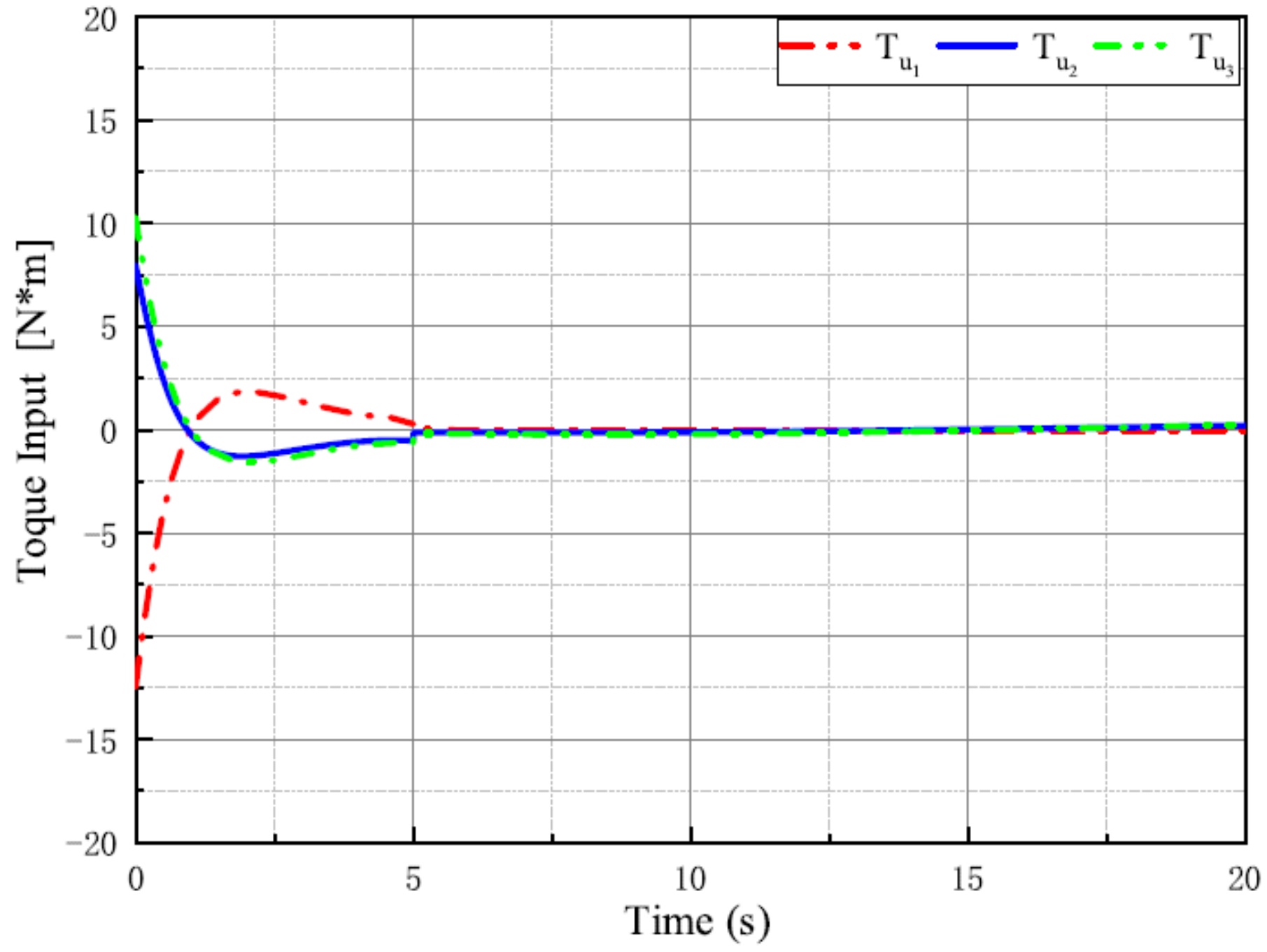

Figure 3

Control input 


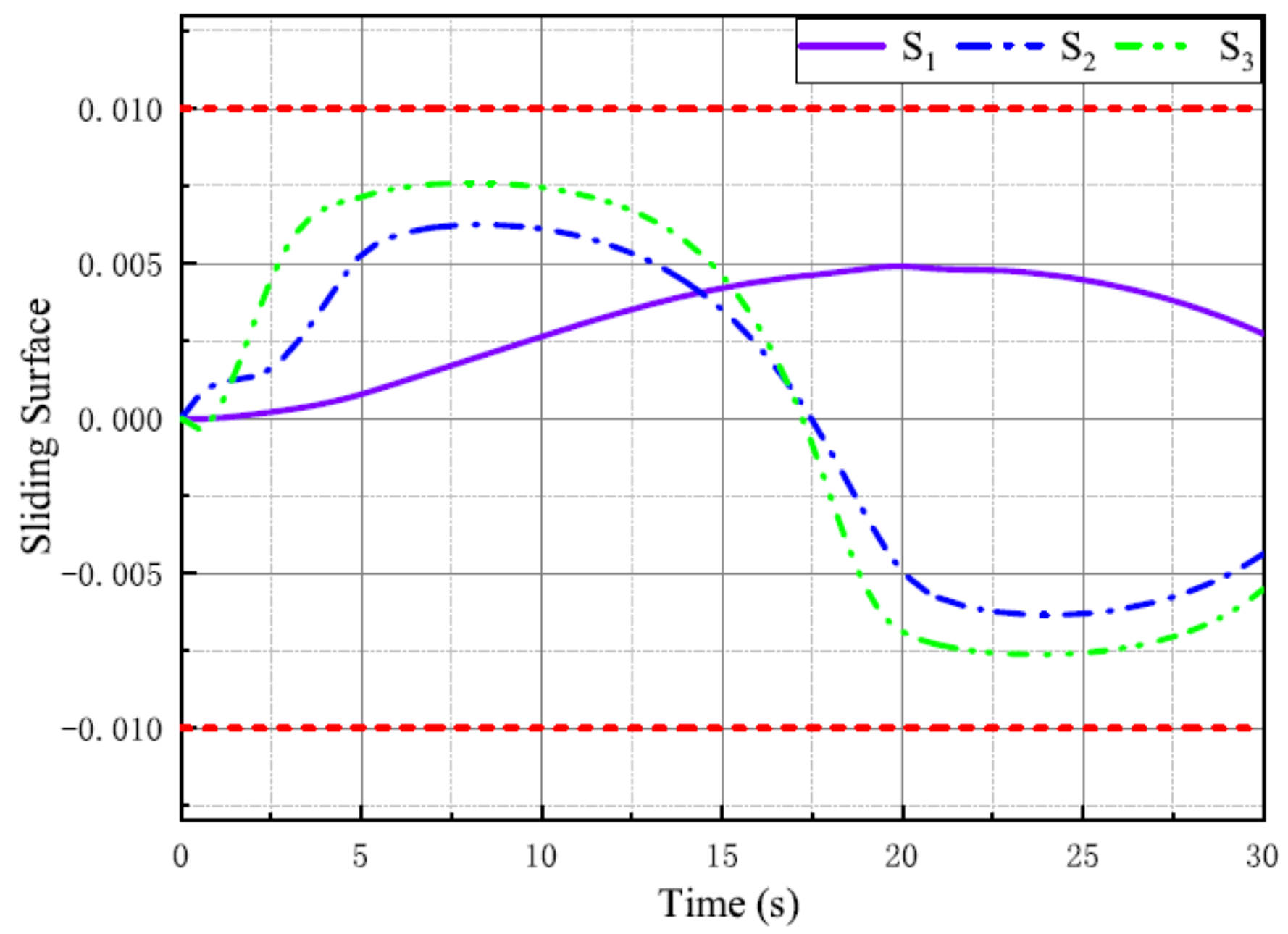

Figure 4

Sliding mode surface function with the PBF 

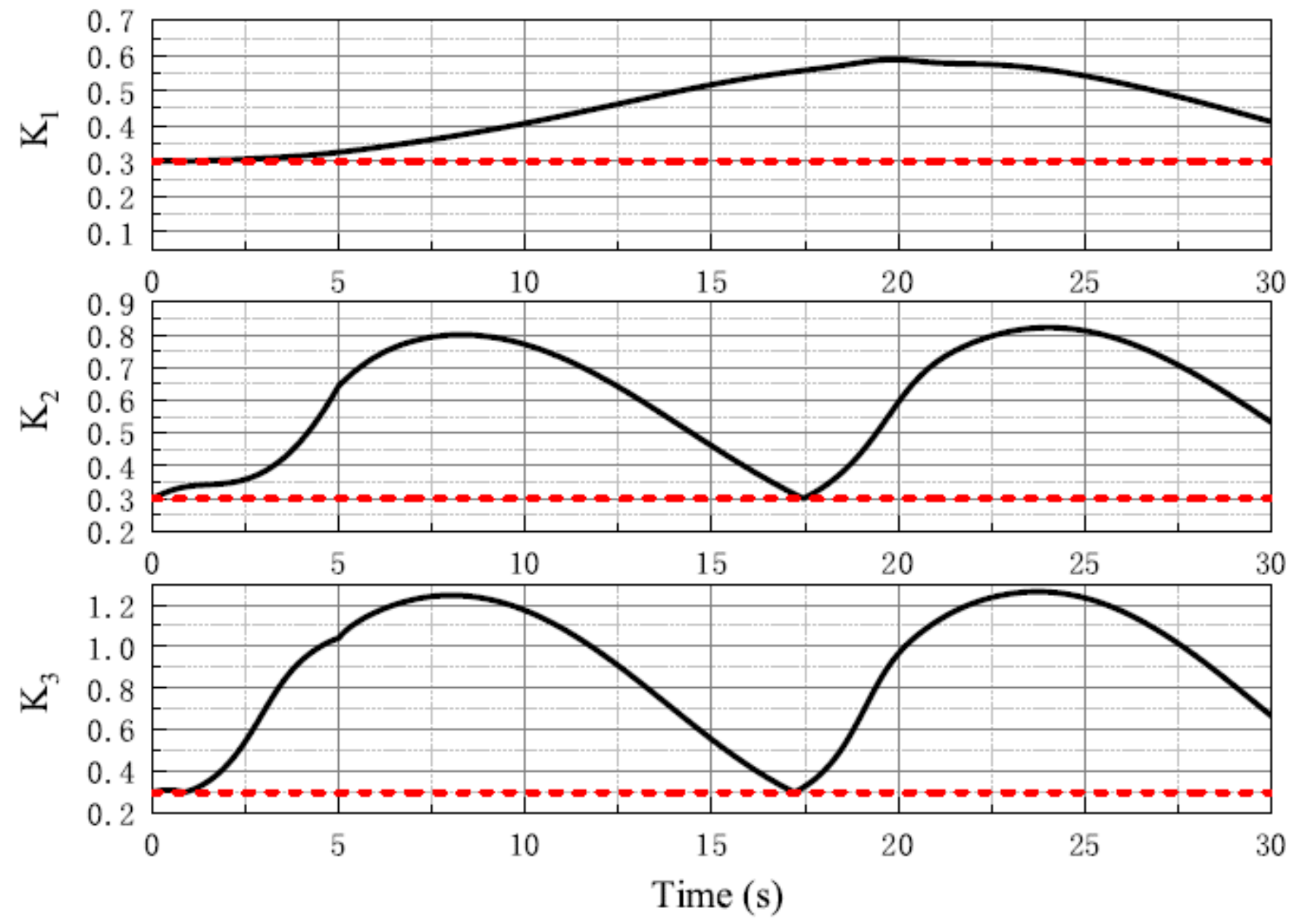

Figure 5

The gain parameter $\mathrm{K}$ with $\mathrm{PBF}$ 


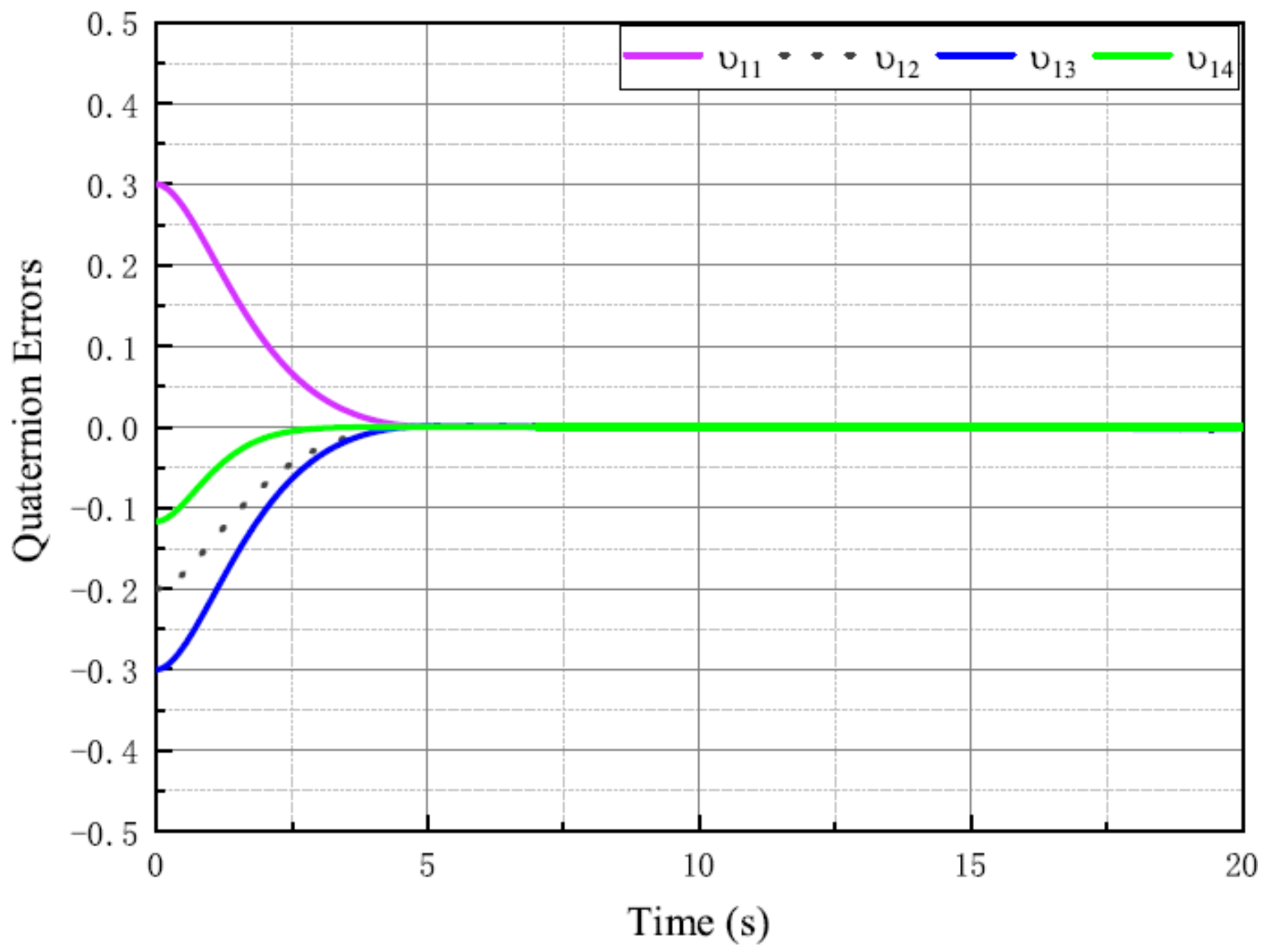

Figure 6

Attitude quaternion tracking errors 


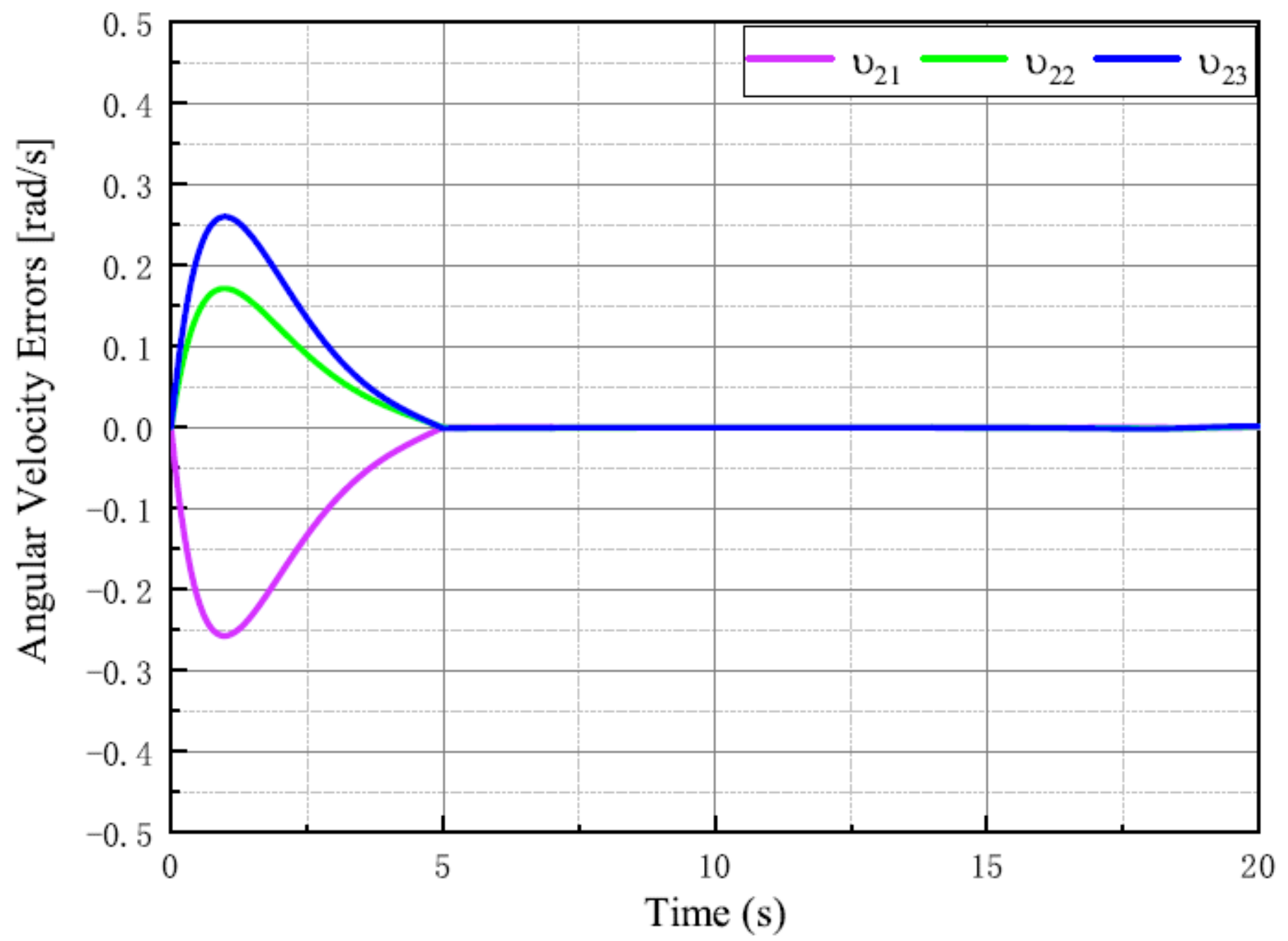

Figure 7

Angular velocity tracking errors 


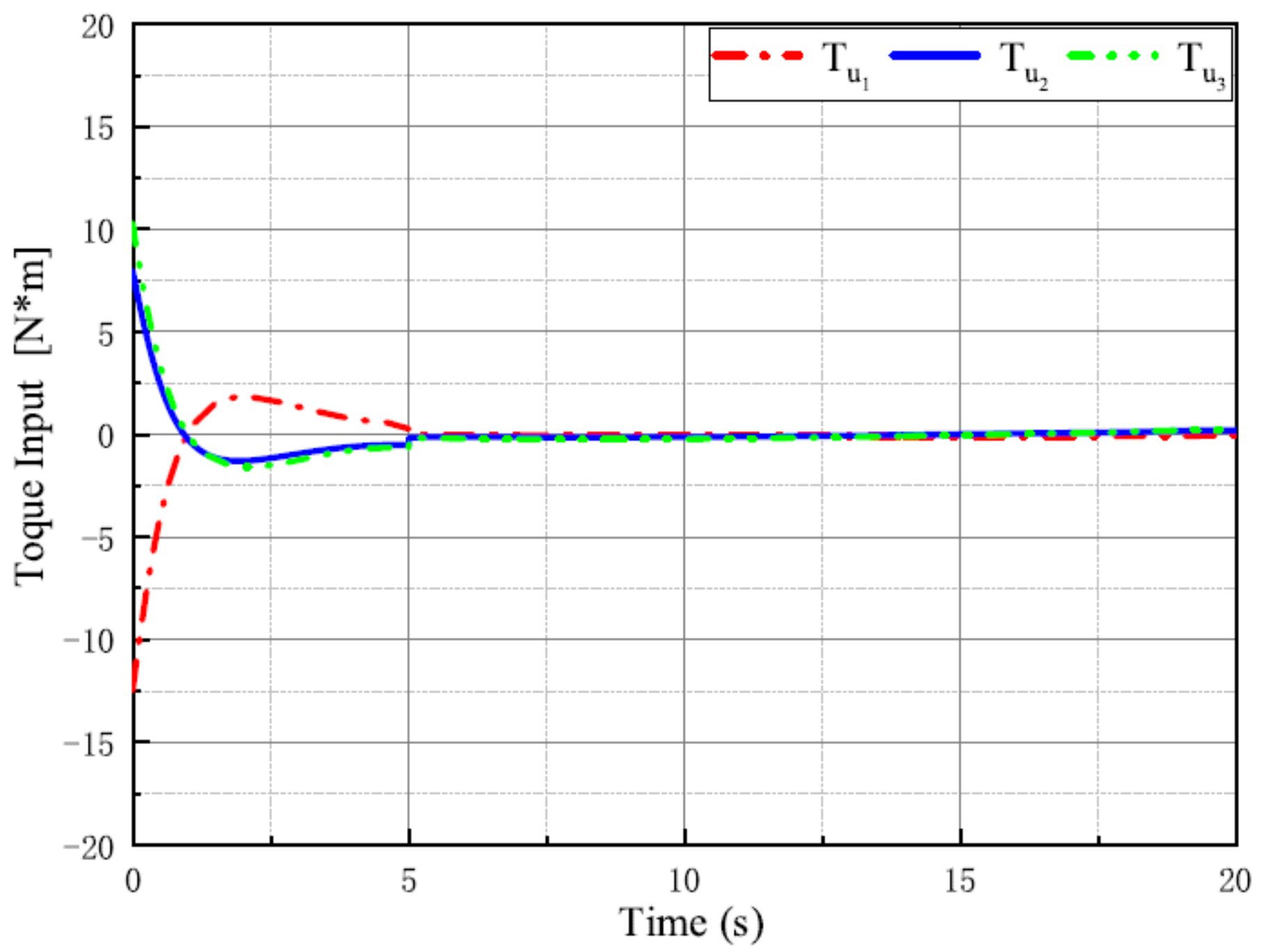

Figure 8

Control input 


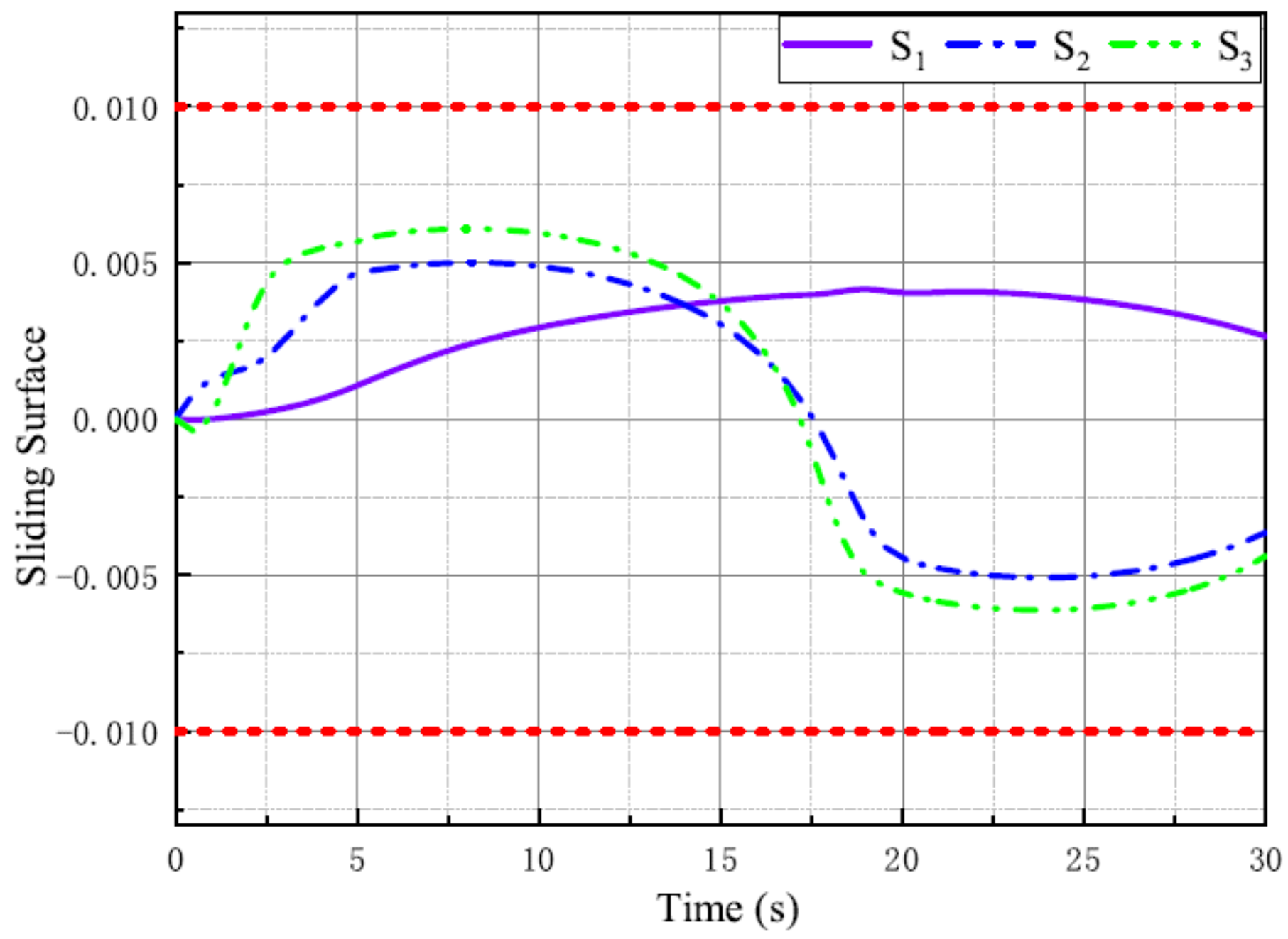

Figure 9

Sliding mode surface function with the PSBF 

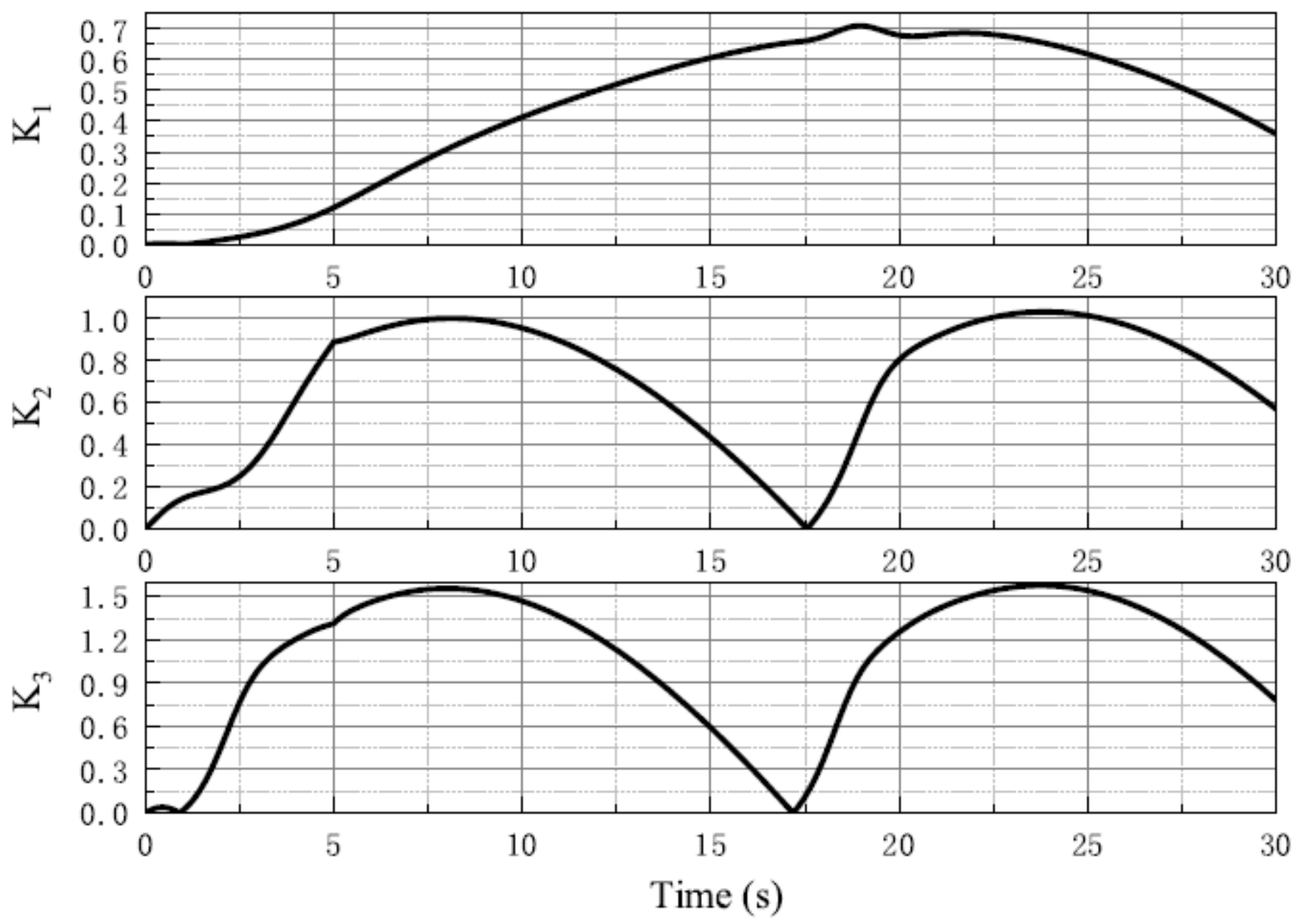

Figure 10

The gain parameter $\mathrm{K}$ with PBF 